\title{
BIFURCATION FOR THE SOLUTIONS OF EQUATIONS INVOLVING SET VALUED MAPPINGS
}

\author{
E.U. TARAFDAR \\ Department of Mathematical Sciences, \\ McMaster University, \\ Hamilton. ONTARIO. \\ CANADA. L8S $4 \mathrm{~K} 1$.

\section{H.B. THOMPSON} \\ Department of Mathematics, \\ University of Queensland, \\ ST. LUCIA. 4067. QUEENSLAND. \\ AUSTRALIA. \\ (Received December 27, 1983)
}

ABSTRACT. This paper is devoted to a generalization of the bifurcation theorem of Krasnosel'skii and Rabinowitz to the set valued situation.

KEY WORDS AND PHRASES. Compact set valued mapping, isoiated zero, index, characteristic value, multiplicity, bifuraation point.

1980 MATHEMATICS SUBJECT CLASSIFICATION CODE. Primary 47H15, 47A50; Seconda!! 47H10, $47 A 55,54 H 25$.

\section{INTRODUCTION.}

In the Leray-Schauder degree theory the study of the index of an isolated fixed point of a compact mapping is a basic tool for the calculation of the degree. A fundamental and well-known result of Leray and Schauder [1], commonly known as the Leray-Schauder principle, is that the topological index at zero of an invertible linear perturbation of the identity I-A in a Banach space can be expressed in terms of multiplicity of the characteristic values of A lying in the open interval $(0,1)$. Krasnosel'skii [2] employed this result as a basic tool to develop his bifurcation theory for equations of the form:

$$
x-\mu A x-R(x, \mu)=0
$$

in a real Banach space $\mathrm{X}$, where $\mathrm{A}: \mathrm{X} \rightarrow \mathrm{X}$ is a linear compact mapping, $\mathrm{R}: \bar{\Omega} \mathrm{x} R \rightarrow \mathrm{X}$ is compact mapping, $\mathrm{R}(\mathrm{x}, \mu)$ is of $\rho(|| \mathrm{x}||)$ uniformly in $\mu$ in compact intervals and $\Omega$ is a bounded open neighbourhood of zero in $x$. Rabinowitz [3] studied the global character of the solution set of such equations and applied his results in many directions. Because of the wide scope of applicability of the bifurcation theorem of crasnosel'skii and Rabinowitz a tremendous research interest in this field has been evidenced 
currently (see Cranda11 and Rabinowitz [4] and the literature cited there).

The aim of this paper is to generalize the theorems of Krasnowel'skii and Rabinowitz to the set valued situation, i.e., to equations of the form:

$$
x \in \mu A x+R(x, \mu)
$$

where $A$ is above, $B: \bar{\Omega} \times R \rightarrow C K(X)$ is a compact set valued mapping and $B$ is of o $(|| x||)$ uniformly in $\mu$ in compact intervals (for definitions see Section 2), $\Omega$ being as above and $C K(X)$ being the set of all compact convex subsets of $X$. From our result for equations of the form (1.2) we have dedured the same result for equations of the form:

$$
L x \in \mu A x+R(x, \mu)
$$

where $\mathrm{L}: \mathrm{X} \rightarrow \mathrm{Z}$ is a linear mapping, $\mathrm{A}: \mathrm{X} \rightarrow \mathrm{Z}$ a compact linear mapping, $\mathrm{B}: \bar{\Omega} \mathrm{x} R \rightarrow \mathrm{CK}(\mathrm{Z})$ a compact mapping and of $o(|| x||)$ uniformly in $\mu$ in compact intervals. The result for the equation when $R$ is single valued was first obtained by Laloux and Mawhin [5] (see also Gaines and Mawhin [6] ). However, even when $R$ is single valued our result is more general than that of Laloux and Mawhin [5] (see remark 4.3).

As an application of our results, we have included a two point boundary value problem for a generalized ordinary differential equation, although we hope that these results will find application to problems in control theory, mathematical economics and related problems. We have made the paper self-contained as far as is practically possible.

2. Throughout the paper $X$ will denote a real Banach space and $C K(X)$ will denote the set of all compact convex subsets of $X$. A set valued mapping $N: \Omega \subset X \rightarrow C K(X)$ is said to be compact if $\mathrm{N}$ is upper semicontinuous and maps bounded subsets of $\Omega$ into relatively compact subsets of $x$. The degree theory which will be used here is due to $\mathrm{Ma}$ [7] although all the results obtained for compact mappings will equally hold for more general mappings known as ultimately compact mappings by applying the degree theory for such mappings due to Petryshyn and Fitzpatrick [8].

INDEX. Let $\Omega$ be an open neighbourhood of a $\epsilon \mathrm{X}$ and $\mathrm{N}: \bar{\Omega}$ - CK $(X)$ be a compact mapping. We assume that $a \in N(a)$ and there exists $\varepsilon_{0}>0$ such that $B_{\varepsilon}$. (a) $\subset \Omega$ and $(I-N)^{-1}(0) \cap B \varepsilon_{0}(a)=\{a: ; i . e ., a$ is an isolated zero of $I-N$, where

$$
B_{\varepsilon_{0}}(a)=\left\{x \in X:|| x-a||<\varepsilon_{0}\right\}
$$

and $I$ is Identity mapping on $X$. This implies that for every

$$
\varepsilon \in\left(0, \varepsilon_{O}\right), 0 \notin(\mathrm{I}-\mathrm{N})\left(\partial \mathrm{B}_{\varepsilon}(\mathrm{a})\right)
$$

where $\partial B_{\varepsilon}(a)$ denotes the boundary of $B_{\varepsilon}(a)$. Thus the degree $d\left(I-N, B_{\varepsilon}(a), 0\right)$ is defined and is independent of $\varepsilon \in\left(0, \varepsilon_{O}\right)$ by the excision property of the degree.

DEFINITION 2.1. Under the above assumptions the index of the fixed point a of $N$ is the integer $d\left(I-N, B_{\varepsilon}(a), 0\right)$ for any $\varepsilon \in\left(0, \varepsilon_{0}\right)$ and will be denoted by $i(I-N, a)$.

REMARK 2.1. If $\mathrm{N}$ is single valued, then the index of a fixed point a of $\mathrm{N}$ as defined above coincides with the Leray-Schauder index.

PROPOSITION 2.1. Let $\mathrm{N}: \bar{\Omega} \rightarrow \mathrm{CK}(\mathrm{X})$ be a compact-mapping such that $0 \notin(\mathrm{I}-\mathrm{N})(\partial \Omega)$ and $(\mathrm{I}-\mathrm{N})^{-1}(0)$ is a finite set, say, $\left\{\mathrm{a}_{1}, \mathrm{a}_{2}, \ldots, \mathrm{a}_{\mathrm{n}}\right\}$; i.e., the set of fixed points of $\mathrm{N}$ is finite. 
Then

$$
\mathrm{d}(\mathrm{I}-\mathrm{N}, \Omega, 0)=\sum_{j=1}^{\mathrm{n}} \mathrm{i}\left(\mathrm{I}-\mathrm{N}, \mathrm{a}_{\mathrm{j}}\right) .
$$

PROOF. We can find positive numbers $\varepsilon_{j}, j=1,2, \ldots, n$ such that

$$
\overline{B_{\varepsilon_{j}}\left(a_{j}\right)} \cap \overline{B_{\varepsilon_{k}}\left(a_{k}\right)}=\phi \text { for any } j, k \in\{1,2, \ldots, n\}
$$

with $j \neq k$.

By the additivity and excision properties of degree we have

$$
d(I-N, \Omega, 0)=d\left(I-N,{ }_{j=1}^{n} B_{\varepsilon_{j}}\left(a_{j}\right), 0\right)=\sum_{j=1}^{n} i\left(I-N, a_{j}\right) \text {. }
$$

DEFINITION 2.2. Let $\Omega \subset \mathrm{X}$ be a bounded open neighbourhood of the origin and $\mathrm{B}: \bar{\Omega} \rightarrow \mathrm{CK}(\mathrm{X})$ be an upper semicontinuous set valued mapping. B is said to be of order $o(|| x||)$ if

$$
\|x\|^{-1} \sup \{\|y\|: y \in B(x)\} \rightarrow 0 \text { as }\|x\| \rightarrow 0 \text {. }
$$

REMARK 2.2. It is clear from the definition that $B(0)=0$ and the definition of o( $\mid\{x \mid 1)$ coincides with the usual definition of $o\left(|| x_{1} \mid\right)$ when $B$ is single valued.

THEOREM 2.1. Let $\mathrm{N}=\mathrm{A}+\mathrm{B}$ where $\hat{\mathrm{A}}: \mathrm{X} \rightarrow \mathrm{X}$ is a linear compact mapping with ier $(\mathrm{I}-\mathrm{A})=\{0\}$ and $\mathrm{B}: \bar{\Omega} \rightarrow \mathrm{CK}(\mathrm{X})$ is a compact mapping of order $\mathrm{o}(\|\mathrm{X}\|)$ where $\Omega \subset \mathrm{X}$ is a bounded open neighbourhood of the origin. Then 0 is an isolated zero of (I-N) and

$$
i(I-N, 0)=i(I-A, 0)
$$

where $i(I-A, 0)$ is the usual Leray-Schauder index of the fixed point 0 of $A$.

PROOF. Since $B$ is of order $O(|| x||)$ and $A$ is linear, $N(0)=0$. We can easily verify that for each $x \in \bar{\Omega}$,

$$
(I-N)(x)=(I-A)\left[I-(I-A)^{-1} B\right](x)
$$

That $(I-A)^{-1}$ exists follows from Riesz theory.

Now from the fact that $B$ is of order $o(|| x||)$ it follows that there exists $\varepsilon_{0}>0$ such that $\bar{B}_{\varepsilon_{0}}(0) \subset \Omega$ and for each $x \in \overline{B_{\varepsilon_{0}}(0)}$,

$$
\sup \left\{\|y\|: y \in(I-A)^{-1} B(x)\right\} \leq \frac{1}{2}|| x|| \text {. }
$$

Hence for each $\varepsilon \in\left(0, \varepsilon_{0}\right]$ and every $(x, \lambda) \in B_{\varepsilon}(0) \times[0,1]$

we have

$$
\begin{aligned}
\inf \{\|x-y\|: y \in \lambda & \left.(I-A)^{-1} B(x)\right\} \geq \inf \left\{\|x\|-\|y\|: y \in \lambda(I-A)^{-1} B(x)\right\} \\
& \geq\|x\|-\sup \left\{\|y\|: y \in \lambda(I-A)^{-1} B(x)\right\} \\
& \geq \frac{i}{2}|| x \| .
\end{aligned}
$$

(2.1) and (2.2) together imply that the only fixed point of $N$ in $B_{f_{0}}(0)$ is 0 ; i.e., $(\mathrm{I}-\mathrm{N})^{-1}(0) \cap \overline{\mathrm{B}}_{\varepsilon_{0}}(0)=\{0\}$.

By the homotopy invariance property

$$
\begin{aligned}
& \mathrm{d}\left(\mathrm{I}-\lambda(\mathrm{I}-\mathrm{A})^{-1} \mathrm{~B}, \mathrm{~B}_{\varepsilon}(0), 0\right)=\mathrm{d}\left(\mathrm{I}, \mathrm{B}_{\varepsilon}(0), 0\right)=1 \\
& \text { for every } \varepsilon \in\left(0, \varepsilon_{0}\right] \text { and } \lambda \in[0,1] \text {. }
\end{aligned}
$$

Now from $(2.1),(2.3)$ and the product theorem of degree we have 


$$
1\left(\left[-N, D_{\varepsilon}(0), n\right)=d\left(I-A, B_{\varepsilon}(0), 0\right) \cdot 1\right.
$$

for each $\varepsilon \in\left(0, \varepsilon_{0}\right]$; i.e.,

$$
i(I-N, 0)=i(I-A, 0) \text {. }
$$

3. CHARACTERISTIC VALUE AND MULTIPLICITY.

Let $A: \because$ - $X$ be a linear compact mapping and $r(A)=\left\{\mu: \mu^{-1}\right.$ is an eigenvalue of $\left.A\right\}$. Each $\lambda \in r(A)$ is called a characteristic value of $A$.

The multiplicity of $\mu \in \mathrm{r}(A)$ is the integer

$$
B(\mu)=\operatorname{dim} \operatorname{ker}\left[[-\mu A]^{n(\mu)}\right. \text {, }
$$

where $n(\mu)$ is the smallest non-negative integer $n$ such that

$$
\operatorname{ker}[I-\mu A]^{n}=\operatorname{ker}[I-\mu A]^{n+1} \text {. }
$$

Since $A$ is compact, $B(\mu)$ is finite.

A real number $\mu$ is said to be a regular value of $A$ if $(I-\mu A)^{-1}$ exists and is continuous.

If $\mu \in \mathrm{R}$ is not a characteristic value of $\mathrm{A}, \mathrm{x}=0$ is an isolated zero of $\mathrm{I}-\mu \mathrm{A}$ and $i_{L S}(I-\mu A, 0)$, the Leray-Schauder index of $I-\mu A$ at zero will be simply denoted by $i(\mu)$.

We write the following well-known Leray-Schauder principle as a lemma.

LEMMA 3.1. If $A: X \rightarrow X$ is a compact linear mapping and $\mu_{1}, \mu_{2}$ with $\mu_{1}<\mu_{2}$ are not characteristic values of $A$, then $i\left(\mu_{1}\right)=(-1)^{B} i\left(\mu_{2}\right)$ where $B$ is the sum of multiplicities of the characteristic values of $A$ lying in the interval $\left[\mu_{1}, \mu_{2}\right]$.

4. BIFURCATION. Throughout the rest of the paper we will assume $\Omega$ to be an open bounded neighbourhood of the origin in $\mathrm{X}$.

DEFINITION 4.1. Let $\mathrm{N}: \bar{\Omega} \rightarrow \mathrm{CK}(\mathrm{X})$ be a set valued mapping satisfying

(a) $\quad \mathrm{N}$ is upper semicontinuous and compact on bounded subsets of $\bar{\Omega} \times \mathrm{R}$;

(oo) for each $\mu \in \mathrm{R}$,

$0 \in \mathrm{N}(0, \mu)$.

Thus for each $\mu \in R, x=0$ is a solution of the equation

$$
\mathrm{x} \in \mathrm{N}(\mathrm{x}, \mu) \text {. }
$$

A point $\left(0, \mu_{0}\right)$ will be said to be a bifurcation point for the solution of the equation (4.1), or simply a bifurcation point of $\mathrm{N}$ if every neighbourhood of $\left(0, \mu_{0}\right)$ contains at least one solution $(x, \mu)$ of the equation (4.1) with $x \neq 0$. By abuse of notation we will sometimes refer to $\mu_{0}$ as the bifurcation point.

LEMMA 4.1. Let $\mathrm{N}$ be as above satisfying (o) and (oo)。 If the interval $\left[\mu_{1}, \mu_{2}\right]$ contains no bifurcation point of $\mathrm{N}$, then there exists a $\delta>0$ such that, for each $\mu \in\left[\mu_{1}, \mu_{2}\right]$ and $\mathrm{x} \in \bar{\Omega} \cap \mathrm{B}_{\delta}(0)$,

$$
x \in N(x, \mu) \Rightarrow x=0 \text { 。 }
$$

PROOF. Let $\mathrm{S}=\left\{(\mathrm{x}, \mu) \epsilon \bar{\Omega} \times\left[\mu_{1}, \mu_{2}\right]: \mathrm{x} \in \mathrm{N}(\mathrm{x}, \mu)\right\}$. From the compactness of $\mathrm{N}$, it follows that $S$ is a compact subset of $X \times R$. We suppose, if possible, that the lemma is not true. Then for each positive integer $n$, there exist $\tilde{\mu}_{n} \epsilon\left[\mu_{1}, \mu_{2}\right], x_{n} \in \bar{\Omega} \cap B_{1 / n}(0)$ such that 


$$
x_{n}=N\left(x_{n}, \tilde{u}_{n}\right) \text { and } x_{n} \neq 0 \text {. }
$$

Now $\left(x_{n}, \tilde{\mu}_{n}\right) \leftarrow S$ which is compact. Hence $\left(x_{n}, \tilde{\mu}_{n}\right)$ has a convergent subsequence converging to $\left(x_{0}, \tilde{\mu}_{n}\right)$. Clearly $x_{0}=0$ and $\left(0, \tilde{\mu}_{0}\right)$ is a bifurcation point of $N$ in $\left[\mu_{1}, \mu_{2}\right\rceil$ which contradicts the hypothesis. Hence the lemma is proved.

THEOREM 4.1. Let $\mathrm{N}$ be as above satisfying (o) and (oo) and let $\mu_{1}, \mu_{2} \div \mathrm{R}$ with $\mu_{1}<\mu_{2}$. Further suppose that $i\left(\mu_{j}\right)=i\left[I-N\left(\cdot, \mu_{j}\right), 0\right], j=1,2$ are defined and $i\left(\mu_{1}\right) \neq$ $i\left(\mu_{2}\right)$. Then there exists $\mu_{0} \cdot\left[\mu_{1}, \mu\right.$. I such that $\left(0, \kappa_{0}\right)$ is a bifurcation point of $\mathrm{N}$.

PROOF. As $i\left(\mu_{j}\right), j=1,2$ are defined, there exist $\delta_{j}>0, j=1,2$ such that $\left[\left(I-N\left(\cdot, \mu_{j}\right)\right)^{-1}(0)\right] \cap B_{\delta_{j}}(0)=\{0\}$.

Suppose that the conclusion of the theorem is false. Then by the above lemma there exists $\delta_{3}>0$ such that for each $\mu \in\left[\mu_{1}, \mu_{2}\right]$ and $x \in B_{\delta_{3}}(0) \cap \bar{\Omega}$,

$$
\mathrm{x} \in \mathrm{N}(\mathrm{x}, \mu)=\mathrm{x}=\boldsymbol{\theta} \text {. }
$$

We set $\delta_{0}=\min \left(\delta_{1}, \delta_{2}, \delta_{3}\right)$. Then for each $\delta \in\left(0, \delta_{0}\right]$, each $\lambda \in[0,1], x \in B_{\delta}(0)$,

$$
x \in N\left(x, \lambda \mu_{1}+(1-\lambda) \mu_{2}\right) \Rightarrow x=0 .
$$

Hence it follows that for each $\delta \in\left(0, \delta_{0}\right], \lambda \in[0,1]$ and $x \in \partial B_{\delta}(0)$.

$$
x \notin N\left(x, \lambda \mu_{1}+(1-\lambda) \mu_{2}\right)
$$

Therefore by Homotopy Invariance Theorem

$$
\begin{aligned}
\mathrm{i}\left(\mu_{1}\right) & =\mathrm{d}\left[\mathrm{I}-\mathrm{N}\left(\cdot, \mu_{1}\right) \mathrm{B}_{\delta}(0), 0\right] \\
& =\mathrm{d}\left[\mathrm{I}-\mathrm{N}\left(\cdot, \mu_{2}\right) \mathrm{B}_{\delta}(0), 0\right]=\mathbf{i}\left(\mu_{2}\right)
\end{aligned}
$$

which contradicts our hypothesis. Hence the theorem is proved.

THEOREM 4.2. Let $N: \bar{\Omega} \times R \rightarrow C K(X)$ be such that

$$
\mathrm{N}(\mathrm{x}, \mu)=\mu \mathrm{Ax}+\mathrm{B}(\mathrm{x}, \mu),
$$

where $A: X \rightarrow X$ is a linear compact (single valued) mapping and $B: \bar{\Omega} \times R \rightarrow X$ is upper semicontinuous and compact on bounded subsets of $\bar{\Omega} \times \mathrm{R}$ with $\mathrm{B}(\mathrm{x}, \mu)=\mathrm{o}(|| \mathrm{x}||)$ uniformly in $\mu$ on compact intervals. Then for each bifurcation point $\left(0, \mu_{0}\right)$ of $N, \mu_{0}$ is a characteristic value of $A$.

PROOF. Again we prove this theorem by contradiction. Suppose that $\left(0, \mu_{0}\right)$ is a bifurcation point of $N$ and $\mu_{0}$ is not a characteristic value. Then $A_{0}=\left(I-\mu_{0} A\right)^{-1}$ exists and is continuous.

Let $F: \bar{\Omega} \times \mathrm{R} \rightarrow \mathrm{X}$ and $\mathrm{G}: \bar{\Omega} \times \mathrm{R} \rightarrow \mathrm{CK}(\mathrm{X})$ be defined as follows:

$$
F(x, \mu)=\left(\mu-\mu_{0}\right) A_{O} A x
$$

and

$$
G(x, \mu)=A_{o} B(x, \mu) \text {. }
$$

Clearly $F$ and $G$ are compact on bounded subsets of $\bar{\Omega} \times R$. Also from the assumption that $B(x, \mu)=o(|| x||)$ and the continuity of $A_{o}$ it follows that $A_{o} B(x, \mu)=o(|| x||)$ uniformly in $\mu$ in compact intervals.

Let $\delta>0$ be such that $\delta|| A_{0} A|| \leq \frac{1}{3}$ and $\rho>0$ be such that whenever 
$(x, \mu) \in B_{\rho}(0) \times\left[\mu_{,}-\delta, \mu_{0}+\delta\right]$ we have

$$
\|\mathrm{x}\|^{-1} \sup \left\{\|\mathrm{y}\|: \mathrm{y} \in \mathrm{A}_{\mathrm{O}} \mathrm{B}(\mathrm{x}, \mu)\right\} \leq \frac{1}{3} \text {. }
$$

Then for each $x \in\left[B_{\rho}(0) \cap \bar{\Omega}\right] \backslash\{0\}$ and $\mu_{L} \leq\left[\mu_{0}-\delta, \mu_{0}+\delta_{1}\right]$

$$
\begin{aligned}
& \inf \left\{|| A_{0} x-y||: y \in A_{0} N(x, u) \mid\right. \\
& =\inf \left\{\mid\left\{A_{0}\left[\left(\left[-u_{0} A\right) x+\nu_{0} A x\right\rfloor-\mu A_{O} A x-v||: v \in A_{O} B(x, w)\right\}\right.\right. \\
& =\inf \left\{|| x+\left(\mu_{0}-\mu\right) A_{O} A x-v \mid \|: v \in A_{O} B(x, \mu)\right\} \\
& \geq|| x||-\left|\mu-\mu_{0}\right||| A_{0} A|||| x||-\sup \left\{|| v||: v \in A_{O} B(x, \mu)\right\} \\
& \geq\left[1-\delta|| A_{0} A||-\frac{1}{3}\right\rfloor|| x|| \geq \frac{1}{3}|| x|| \text {. }
\end{aligned}
$$

Hence there exist $n>0$ and $\delta>0$ such that for each

$$
(x, \mu) \in\{[B,(0) \cap \bar{\Omega}]:\{0\}\} \times\left[\mu_{0}-\delta, \mu_{0}+\delta\right], x \notin N(x, \mu) .
$$

sut this implies that $\mu$ is not a bifurcation point, which is a contradiction. Thus the theorem is proved.

The following theorem in the single valued case is due to Krasnosel'skii [2].

THEOREM 4.3. Let $\mathrm{N}: \bar{\Omega} \times \mathrm{R} \rightarrow \mathrm{CK}(\mathrm{X})$ be given by $\mathrm{N}(\mathrm{x}, \mu)=\mu \mathrm{Ax}+\mathrm{B}(\mathrm{x}, \mu)$ where $\mathrm{A}$

and $B$ are as in Theorem 4.2. If $\mu_{0}$ is a characteristic value of $A$ of odd multiplicity $B_{O}$, then $\left(0, \mu_{0}\right)$ is a bifurcation point of $\mathrm{N}$.

PROOF. Since A is compact, $\mu_{0}$ is an isolated characteristic value of $A$. Hence there exists $\varepsilon>0$ such that $\mu_{0}$ is the only characteristic value of $A$ in $\left[\mu_{0}-\varepsilon, \mu_{0}+\varepsilon\right]$. Thus from Lemma 3.1 we have

$$
\begin{aligned}
i\left(\mu_{0}-\varepsilon\right) & =i\left[I-\left(\mu_{0}-\varepsilon\right) A, 0\right]=(-1)^{\beta} \mathrm{i}\left[I-\left(\mu_{0}+\varepsilon\right) A, 0\right] \\
& =(-1)^{\beta} i\left(\mu_{0}+\varepsilon\right) .
\end{aligned}
$$

Hence from the above equality and Theorem 2.1 we have

$$
\mathrm{i}\left[\mathrm{I}-\mathrm{N}\left(\cdot, \mu_{0}-\varepsilon\right), 0\right]=(-1)^{\beta_{0}} \mathrm{i}\left[\mathrm{I}-\mathrm{N}\left(\cdot, \mu_{0}+\varepsilon\right), 0\right] \text {. }
$$

Now since $B_{O}$ is odd, we have

$$
i\left[I-N\left(\cdot, \mu_{0}-\varepsilon\right), 0\right] \neq i\left[I-N\left(\cdot, \mu_{0}+\varepsilon\right), 0\right] \text {. }
$$

Hence by Theorem 4.1 there exists a bifurcation point $(0, \bar{\mu})$ of $N$ with $\bar{\mu} \epsilon\left[\mu_{0}-\varepsilon, \mu_{0}+\varepsilon\right]$. But since $\mu_{0}$ is the only characteristic value in $\left[\mu_{c}-\varepsilon, \mu_{0}+\varepsilon\right]$, Theorem 4.2 implies that $\bar{\mu}=\mu_{0}$. Thus we have proved that $\left(0, \mu_{0}\right)$ is a bifurcation point of $\mathrm{N}$.

We note that all Theorems proved above hold with the same proof if we replace $\Omega$ by $\mathrm{X}$.

Let $\mathrm{E}$ denote the space $\mathrm{X} \times \mathrm{R}$ or $\bar{\Omega} \times \mathrm{R}$ where $\Omega$ is a bounded open neighbourhood of the origin in $\mathrm{x}$.

The following global version in the single valued case is due to Rabinowitz [3].

THEOREM 4.4 Let $N: F \rightarrow C K(X)$ be such that $N(x, \mu)=\mu A x+B(x, \mu)$ where $A$ and $B$ are as in Theorem 4.2 with $\bar{\Omega} \times \mathrm{R}$ being replaced by $E$. Let $\mu_{0}$ be a characteristic value of $A$ of odd inultiplicity. Let $S$ denote the closure of all nontrivial solutions of (4.1). Then $S$ contains a component $C$ (i.e., a maximal $c$ losed and connected subset) which contains $\left(0, \mu_{0}\right)$ and either is unbounded or contains $(0, \tilde{\mu})$ where $\tilde{\mu}$ is a characteristic value of $A$ 
and $\tilde{\mu} \neq \mu_{0}$.

The proof of this theorem is exactly similar to that given by Rabinowitz [3] (also see Crandall and Rabinowitz [4] ) for the single valued case. However we include the proof for the sake of completeness. We will need the following two lemmas.

LEMMA 4.2. (Whyburn (1958)). Let $\mathrm{K}$ be a compact metric space and $\mathrm{A}$ and $\mathrm{B}$ two disjoint closed subsets of $K$. Then either there is a subcontinuum (i.e., a closed and connected subset) of $\mathrm{K}$ meeting both $\mathrm{A}$ and $\mathrm{B}$, or there exist disjoint compact subsets $K_{A} \supset \Lambda$ and $K_{B} \supset B$ such that $K=K_{A} \cup K_{B}$.

LEMMA 4.3. Under the hypothesis of Theorem 4.4 assume that there exists no subcontinuum $C$ of $S \cup\left\{\left(0, \mu_{0}\right)\right\}$ such that either (i) $C$ is unbounded, or (ii) C contains $(0, \bar{\mu})$ with $\mu_{0} \neq \bar{\mu} \in \mathrm{r}(\mathrm{L})$. Then there exists a bounded open set $\theta$ in $\mathrm{E}$ such that

$$
\left(0, \mu_{0}\right) \in \theta, \partial \theta \cap S=\phi
$$

and

$$
\theta \cap(\{0\} \times R)=\{0\} \times\left(\mu_{0}-\delta, \mu_{0}+\delta\right)
$$

for some positive $\delta$.

PROOF. The proof is similar to that of Lemma 1.2. Let $C_{\mu_{0}}$ be the component of $\left(0, \mu_{0}\right)$ in $S \cup\left\{\left(0, \mu_{0}\right)\right\}$. Then $C_{\mu_{0}}$ is bounded, by $(i)$. Hence by the upper semicontinuity and compactness of $\mathrm{N}, \mathrm{C}_{\mu_{0}}$ is a compact. Let $\mathrm{U}_{\delta}$ be a $\delta$-neighbourhood of $\mathrm{C}_{\mu_{0}}$. Now from the fact that for $\lambda \notin \mathrm{r}(\mathrm{L})$, by Theorem $2.1,(0, \lambda)$ is an isolated solution of $(4.1)$ and from (ii) it follows that for $0<\delta<\varepsilon_{0}$ sufficiently small $U_{\delta}$ contains no solution $(0, \lambda)$ with $\left|\lambda-\mu_{0}\right|>\delta$. Now since $S$ is locally compact, it follows that $K=\bar{U}_{\delta} \cap S$ is compact in the relative topology induced from $E$. Also $C_{\mu_{0}} \cap \partial U_{\delta}=\phi$. Let $K_{1}=C_{\mu_{0}}$ and $K_{2}=$ $\left(\partial \mathrm{U}_{\delta}\right) \cap \mathrm{S}$. There is no subcontinuum meeting $\mathrm{K}_{1}$ and $\mathrm{K}_{2}$ for otherwise $C_{\mu_{0}}$ will not be a component. Hence by Lemma 4.2 there exist compact subsets $A \supset K_{1}$ and $B \supset K_{2}$ such that $\mathrm{K}=\mathrm{A} \cup \mathrm{B}$. Let $\theta$ be an $\varepsilon$-neighbourhood of $\mathrm{A}$ where $\varepsilon$ is less than the distance between $\mathrm{A}$ and $B$. We can easily see that $A$ fulfills the demand of the lemma.

PROOF OF THEOREM 4.4. Let $\mathrm{C}_{\mu_{0}}$ be the component of $\left(0, \mu_{0}\right)$ in $\left.S \cup\left\{0, \mu_{0}\right)\right\}$. Assume that the theorem is false. Then by Lemma 4.3 there is a bounded open set 0 such that

$$
\mathrm{C}_{\mu_{0}} \subset \theta, \partial \theta \cap \mathrm{S}=\phi
$$

and

$$
\theta \cap(\{0\} \times R) \subset\{0\} \times\left(\mu_{0}-\delta, \mu_{0}+\delta\right)
$$

for some $\delta>0$. Let $\theta_{\lambda}=\{\mathrm{x} \in \mathrm{x}:(\mathrm{x}, \lambda) \in \theta\}$. Now for $\lambda$ satisfying $0<\left|\lambda-\mu_{0}\right| \leq \delta,(0, \lambda)$ is an isolated solution of (4.1) (by construction of $\theta$ and Theorem 2.1). Hence there exists $\rho(\lambda)>0$ such that $(0, \lambda)$ is the only solution of $(4.1)$ in $\overline{\mathrm{B}}_{\rho}(\lambda) \times\{\lambda\}$. For $\lambda>\mu_{0}+\delta$ we choose $\rho(\lambda)=\rho\left(\mu_{0}+\delta\right)$ and for $\lambda<\mu_{0}-\delta$ we choose $\rho\left(\mu_{0}-\delta\right)$. By choosing $\rho\left(\mu_{0} \pm \delta\right)$ sufficiently small, we can assume that $\overline{\mathrm{B}}_{\rho}(\lambda)^{\cap} \bar{\theta}_{\lambda}=\phi$ for $\lambda$ satisfying $\left|\lambda-\mu_{0}\right| \geq \delta$. Thus for $\lambda \neq \mu_{0}$ there is no solution of (4.1) in $\partial\left[\theta_{\lambda}-\bar{B}_{\rho}(\lambda)\right] \times\{\lambda\}$ and therefore $\mathrm{d}\left(\mathrm{I}-\mathrm{N}(\cdot, \lambda), \theta_{\lambda}-\overline{\mathrm{B}}_{\rho}(\lambda), 0\right)$ is well defineci or each $\lambda \neq \mu_{0}$. By homotopy invariance $d\left(I-N(\cdot, \lambda), \theta_{\lambda}-\bar{B}_{\rho}(\lambda), 0\right)=$ constant for all $\lambda>\mu_{0}$. However (4.1) has no soltion in $\theta_{\lambda}-\bar{B}_{\rho}(\lambda)$. Hence for $\lambda>\mu_{0}$ we have

$$
\text { (A) } \quad d\left(I-N(\cdot, \lambda), \theta_{\lambda}-\bar{B}_{\rho}(\lambda), 0\right)=0 \text {. }
$$


imilarly (A) holds for $\lambda \leq \mu_{0}$. Moreover, by homotopy invariance we have

$$
\text { (B) } d\left(I-N(\cdot, \lambda), \theta_{\lambda}, 0\right)=\text { constant }
$$

for $\left|\lambda-\mu_{1}\right|<\delta$. Let

$$
\mu_{u}-\delta<\underline{\mu}<\mu_{0}<\bar{\mu}<\mu_{0}+\delta .
$$

Then from

$$
\theta_{\bar{\mu}}=B_{\rho}(\bar{\mu}) \cup\left(\theta_{\bar{\mu}}-B_{\rho}(\bar{\mu})\right)
$$

and by additivity of degree we have

$$
\begin{aligned}
\mathrm{d}\left(\mathrm{I}-\mathrm{N}(\cdot, \bar{\mu}), \theta_{\bar{\mu}}, 0\right) & =\mathrm{d}\left(\mathrm{I}-\mathrm{N}(\cdot, \bar{\mu}), \mathrm{B}_{\rho}(\bar{\mu}), 0\right)+\mathrm{d}\left(\mathrm{I}-\mathrm{N}(\cdot, \bar{\mu}), \theta \bar{\mu}-\overline{\mathrm{B}}_{\rho}(\bar{\mu}), 0\right) \\
& =\mathrm{d}\left(\mathrm{I}-\mathrm{N}(\cdot, \bar{\mu}), \mathrm{B}_{\rho}(\bar{\mu}), 0\right) \text { by }(\mathrm{A}) .
\end{aligned}
$$

Similarly, we can show that

$$
d\left(I-N(\cdot, \underline{\mu}), \theta_{\underline{\mu}}, 0\right)=d\left(I-N(\cdot, \underline{\mu}), B_{\rho}(\underline{\mu}), 0\right) .
$$

Hence by using (B) we obtain

$$
\text { (C) } \quad d\left(I-N(\cdot, \underline{\mu}), B_{\rho}(\mu), 0\right)=d\left(I-N(\cdot, \bar{\mu}), B_{\rho}(\bar{\mu}), 0\right) .
$$

We now define the homotopy

$$
\hat{N}(x, \lambda, t)=\lambda A x+t B(x, \lambda), 0 \leq t \leq 1,
$$

As $B(x, \lambda)$ is of $o(|| x||)$, we can choose $\rho(\vec{\mu})$ sufficiently small to obtain

$$
0 \notin(I-\hat{N})(x, \bar{\mu}, t)
$$

for any

$$
(x, t) \in \partial B_{\rho}(\bar{\mu}) \times[0,1] .
$$

Thus by homotopy invariance

$$
\begin{aligned}
& \mathrm{d}\left(\mathrm{I}-\mathrm{N}(\cdot, \bar{\mu}), \mathrm{B}_{\rho}(\bar{\mu}), 0\right)=\mathrm{d}\left(I-\hat{\mathrm{N}}(\cdot, \bar{\mu}, \mathrm{t}), \mathrm{B}_{\rho}(\bar{\mu}), 0\right) \\
& =\mathrm{d}\left(\mathrm{I}-\hat{\mathrm{N}}(\cdot, \bar{\mu}, 0), \mathrm{B}_{\rho(\bar{\mu})}, 0\right)=\mathrm{d}\left(I-\bar{\mu} \mathrm{A}, \mathrm{B}_{\rho(\bar{\mu}), 0) .}\right.
\end{aligned}
$$

By repeating the same argument for $\underline{\mu}$ and using (C) we obtain

$$
\mathrm{d}\left(I-\underline{\mu} \mathrm{A}, \mathrm{B}_{\rho}(\underline{\mu}), 0\right)=\mathrm{d}\left(\mathrm{I}-\bar{\mu} \mathrm{A}, \mathrm{B}_{\rho}(\bar{\mu}), 0\right)
$$

i.e.,

$$
i(I-\underline{\mu} A, 0)=i(I-\bar{\mu} A, 0)
$$

which is a contradiction in view of the fact that $\mu_{0}$ is a characteristic value of odd multiplicity and in view of Lemma 3.1. Thus the theorem is proved.

REMARK 4.1. Results similar to Theorems $1.16,1.25,1.27,1.40$ in Rabinowitz [3] can also be proved in the set valued case. We have omitted these as their proofs are only repetition of the arguments given by Rabinowitz.

Let $X$ and $Z$ be real Banach spaces. Let us now consider the equation

$$
\operatorname{Lx} \in \lambda A x+B(x, \lambda)=N(x, \lambda)
$$


where $\mathrm{L}: \mathrm{X} \rightarrow \mathrm{Z}$ is a continuous linear mapping, $\mathrm{A}: \mathrm{X} \rightarrow \mathrm{Z}$ is a compact linear mapping and $B: \bar{\Omega} \times R \rightarrow C K(Z)$ is a set valued mapping which is upper semicontinuous and is compact on bounded subset of $\bar{\Omega} \times \mathrm{R}$. We also assume that $0 \in B(0, \lambda)$ for all $\lambda \in R$.

A point $\left(0, \mu_{0}\right)$ is said to be a bifurcation point of the equation (4.2) if every neighbourhood of $\left(0, \mu_{0}\right)$ contains at least one solution $(x, \mu)$ of $(4.2)$ with $x \neq 0$.

THCORFM 4.5. Let $\mathrm{L}, \mathrm{A}$ and $\mathrm{B}$ be as above. Let $\mathrm{B}(\mathrm{x}, \lambda)$ be $o(|| x||)$ uniformly in $\lambda$ in compact intervals; that is,

$$
\|x\|^{-1} \sup \{\|y\|: y \in B(x, \lambda)\} \rightarrow 0 \text { as }\|x\| \rightarrow 0
$$

uniformly in $\lambda$ in compact intervals. Assume that there exists $\bar{\mu} \epsilon$ such that $(L-\bar{\mu} A)^{-1}$ exists and is continuous. Let $\left(\mu_{0}-\bar{\mu}\right)$ be a characteristic value of the compact operator

$$
A_{0}=(L-\bar{\mu} \Lambda)^{-1} A
$$

of odd multiplicity $\beta_{0}$. Then $\mu_{0}$ is a bifurcation point of the equation (4.2).

PROOF. Let us consider the equation

$$
\mathbf{x} \in \lambda \mathrm{A}_{\mathrm{o}} \mathbf{x}+(\mathrm{L}-\bar{\mu} \mathrm{A})^{-1} \mathrm{~B}(\mathbf{x}, \lambda+\bar{\mu})=\overline{\mathrm{N}}(\mathbf{x}, \lambda) \text {. }
$$

Now $(x, \mu)$ is a solution of $(4.2)$ if and only if $(x, \mu-\bar{\mu})$ is a solution of (4.3). Indeed,

$$
\begin{aligned}
& \mathrm{Lx} \in \mathrm{N}(\mathrm{x}, \mu)=\mu \mathrm{Ax}+\mathrm{B}(\mathrm{x}, \mu) \Leftrightarrow \\
& \mathrm{Lx}-\bar{\mu} \mathrm{Ax} \in(\mu-\bar{\mu}) \mathrm{Ax}+\mathrm{B}(\mathrm{x}, \mu-\bar{\mu}+\bar{\mu}) \Leftrightarrow \\
& \mathrm{x} \in(\mu-\bar{\mu})(\mathrm{L}-\bar{\mu} \mathrm{A})^{-1} \mathrm{Ax}+(\mathrm{L}-\bar{\mu} \mathrm{A})^{-1} \mathrm{~B}(\mathrm{x}, \mu-\bar{\mu}+\bar{\mu}) \Leftrightarrow \\
& \mathrm{x} \in(\mu-\bar{\mu}) \mathrm{A}_{\mathrm{O}} \mathrm{x}+(\mathrm{L}-\bar{\mu} \mathrm{A})^{-1} \mathrm{~B}(\mathrm{x}, \mu-\bar{\mu}+\bar{\mu}) \\
& =\overline{\mathrm{N}}(\mathrm{x}, \mu-\bar{\mu}) .
\end{aligned}
$$

Noting that $(L-\bar{\mu} A)^{-1} B(x, \lambda+\bar{\mu})$ is of $o(\|x\|)$ uniformly in $\lambda$ in compact intervals, by applying Theorem 4.3 we conclude that $\mu_{0}-\bar{\mu}$ is a bifurcation point of (4.3). Hence it follows from (4.4) that $\mu_{0}$ is a bifurcation point of $(4.2)$.

REMARK 4.2. The corresponding global version of Theorem 4.5 also holds.

REMARK 4.3. It is clear that in Theorem 4.5 instead of assuming $A$ and $B(x, \lambda)$ to be compact it would suffice to assume $A_{O}=(L-\bar{\mu} A)^{-1} A$ and $(L-\bar{\mu} A)^{-1} B$ to be compact. If we do this, then even when $B$ is single valued our Theorem will be more general than that proved in Laloux and Mawhin [5] in the sense that we are not assuming $L$ to be a Fredholm mapping of index zero. Note that all the conditions in Theorem 4.5 have also been assumed implicitly by Laloux and Mawhin.

5. APPLICATION. Let $\mathrm{CK}(\mathrm{X})$ be as defined in Section 2. Let

$$
F:[0, \pi] \times R \times R^{n} \times R^{n} \rightarrow C K\left(R^{n}\right)
$$

be upper semicontinuous and $q \in L^{1}[0, \pi]$ (abbreviated as $L^{1}$ ) be such that

$$
\begin{aligned}
& \sup \left\{\|u\|: u F(t, \lambda, y, z), \text { some } \lambda \in R, y, z \in R^{n}\right\} \leq q(t) \\
& \forall t \in[0, \pi] .
\end{aligned}
$$

We consider the two point boundary value problem 


$$
\begin{gathered}
\ddot{y}(t) \epsilon \lambda y(t)+F(t, \lambda, y(t), \dot{y}(t)), \text { a.e. } t \epsilon[0, \pi] \\
y(0)=0=y(\pi)
\end{gathered}
$$

where a solution $y:[0, \pi] \rightarrow \mathrm{R}^{\mathrm{n}}$ satisfies $\hat{\mathrm{y}}$ is absolutely continuous on $[0, \pi]$ and $\mathrm{y}$ satisfies $(5.2),(5.3)$.

We assume that for $t \in[0, \pi], \lambda \in R, y, z \in R^{n}$

$$
\frac{\sup \{\|u\|: u \in F(t, \lambda, y, z,)\}}{\|y\|+\|z\|} \rightarrow 0
$$

uniformly for $t \in[0, \pi]$ and $\lambda$ bounded, as ||$y||+\|z\| \rightarrow 0$. For $y, z \in(c[0, \pi])^{n}$ (abbreviated as $\left.(C)^{n}\right)$ let

$$
B(y, z, \lambda)=\left\{f: f:[0, \pi] \rightarrow R^{n} \text { measural) } 1 e, f(t) \in F(t, \lambda, y(t), z(t))\right\} ;
$$

when $z=\frac{d y}{d t}$ we abbreviate this to $B(y, \lambda)$. For

$$
\lambda \in \mathrm{R}, \mathrm{y}, \mathrm{z} \in(\mathrm{C})^{\mathrm{n}}
$$

let

$$
H(t)=F(t, \lambda, y(t), z(t))
$$

then

$$
\mathrm{H}:[0, \pi] \rightarrow \mathrm{CK}\left(\mathrm{R}^{\mathrm{n}}\right)
$$

is upper semicontinuous as a composition of an upper semicontinuous function with a continuous function and hence

$$
\left\{(t, u,(t)) \in[0, \pi] \times R^{n}: u(t) \in H(t) \forall t \in[0, \pi]\right\}
$$

is closed and hence measurable. Thus the set $B(y, z, \lambda)$ is non empty by the results stated in Rockafeller [9] Also $B$ is convex valued as $F$ is convex valued. Since $f \in B(y, z, \lambda)$, $f$ is measurable and

$$
\|f(t)\| \leq q(t) \forall t \in[0, \pi]
$$

su $f \in\left(L^{1}\right)^{n}$.

Let

$$
\mathrm{G}:[0, \pi] \times[0, \pi] \rightarrow \mathrm{R}
$$

be given by

$$
G(x, t)= \begin{cases}\frac{(\pi-x) t}{\pi}, & 0 \leq t \leq x \leq \pi \\ \frac{x(\pi-t)}{\pi}, & 0 \leq x \leq t \leq \pi\end{cases}
$$

and let $K:\left(L^{1}\right)^{n} \rightarrow X$ be given by

$$
K \phi(x)=\int_{0}^{\pi} G(x, t) \phi(t) d t
$$

where

$$
\phi(t)=\left(\phi^{1}(t), \ldots, \phi^{n}(t)\right), \phi^{i} \in L^{1}, 1 \leq i \leq n,
$$


and

$$
x=\left(c^{1}[0, \pi]\right)^{n}
$$

For $S \subseteq\left(L^{1}\right)^{n}$ let

$$
\mathrm{KS}=\{\mathrm{K} \phi: \phi \in \mathrm{S}\} .
$$

For $\mathrm{y} \in \mathrm{X}$ let

$$
N(y, \lambda)=\lambda K v+K B(y, \lambda) .
$$

Thus

$$
\mathrm{N}(\mathrm{y}, \lambda) \in \mathrm{CK}(\mathrm{X})
$$

and finding a solution to problems $(5.2),(5.3)$ is equivalent to finding a solution to

$$
y \in N(y, \lambda)=\lambda K y+K B(y, \lambda) \text {. }
$$

Now

$$
\mathrm{KB}(\mathrm{y}, \lambda) \in \mathrm{CK}(\mathrm{X}) \text { and } \mathrm{KB}: \mathrm{X} \times \mathrm{R} \rightarrow \mathrm{CK}(\mathrm{X})
$$

is upper semicontinuous. This can be seen as follows. As $B$ is convex valued and $K$ is linear $K B$ is convex valued. Since $f \in B(y, \lambda)$ implies $f \in\left(L^{1}\right)^{n}$ and

$$
\|f(t)\| \leq q(t) \forall t \in[0, \pi],\|(\ddot{l i f})(t)\|=|| f(t) \| \leq q(t),
$$

a.e. $t \in[0, \pi]$ so $K B(y, \lambda)$ is equicontinuous in $X$. If $f_{i} \in B(y, \lambda)$ and $\mathrm{Kf}_{\mathbf{i}} \rightarrow g$ in $x$ then

$$
|| f_{i}(t) \| \leq q(t)
$$

so $f_{i} \rightarrow f$ weakly in $\left(L^{1}\right)^{n}$. Thus there exists $h_{i} \epsilon\left(L^{1}\right)^{n}, h_{i}$ convex combinations of the $f_{j}$ such that $h_{i} \rightarrow f$ in $\left(L^{1}\right)^{n}$. Thus $h_{i} \in B(y, \lambda)$ and $h_{j_{k}} \rightarrow f$, a.e. $t \in[0, \pi]$ some subsequence $h_{i_{k}}$. Thus as $F$ is upper semicontinuous $f \in B(y, \lambda)$ and

$$
g=\operatorname{Kf} \epsilon \operatorname{KB}(y, \lambda)
$$

is closed and hence compact. A similar argument using the upper semicontinuity of $F$ with respect to $(\lambda, y, z)$ shows $K B: X \times R \rightarrow C K(X)$ is upper semicontinuous. Now $K: X \rightarrow X$ is completely continuous with eigenvalues

$\mathrm{n}=1,2,3, \ldots$ all of multiplicity one.

$$
\frac{1}{\lambda}=\frac{1}{n^{2}},
$$

From (5.4) we see that for $\lambda \in R$ and $y \in X$

$$
\frac{\sup \{\|u\|: u \in K B(y, \lambda)\}}{\|y\|} \rightarrow 0
$$

as $y \rightarrow 0$ in $x$; here $\|u\|$ and $\|y\|$ are the $x$ norms of $u$ and $y$ respectively. Thus the conditions of Theorem 4.2 are satisfied and the points $\left(0, n^{2}\right)$ are bifurcation points and the problem $(5,2)$, $(5,3)$ has nontrivial solutions $(y, \lambda)$ near $\left(0, n^{2}\right)$.

REMARK 5.1. The upper semicontinuity of $F$ can be relaxed to 
(i) $F(t, \cdot, \cdot, \cdot)$ is upper semicontinuous for almost every $t \in[0, \pi]$

(ii) $F(\cdot, \lambda, y, z$,$) is measurable for all (\lambda, y, z) \in R \times R^{n} \vee \mathbb{R}^{n}$

(iii) F is closed convex valued

and

$$
\text { (iv) for each }(\lambda, y, z) \in R \times R^{n} \times R^{n}
$$

there exists $\mathrm{f}:[0, \pi] \rightarrow \mathrm{R}^{\mathrm{n}}$ measurable such that

$$
f(t) \in F(t, \lambda, y, z)
$$

for all $t \in[0, \pi]$ and there exists fixed $q \in L^{1}$ such that

$$
|| f(t)|| \leq q(t)
$$

for almost every $t \in[0, \pi]$.

Note. The permanent address of the first author is: Department of Mathematics, University of Queensland, St. Lucia, Queensland, Australia, 4067.

\section{REFERENCES}

1. IERAY, J., and SCHAJTER, J., Topologie et équrations functionelles. Ann. Scole Norm. Sup. 51 (1934) 45-78.

2. KRASNOSEL'SKII, M.A., Topological Methods in the Theory of Nonlinear Integral Equations. Pergamon Press, 1963.

3. RABINOWITZ, P.H., Some Global Results for Nonlinear Eigenvalue Problems. J. Funct. Ana1. 7 (1971) 487-513.

4. CRANDALI, M.G., and RABINOWITZ, Paul H., Mathematical Theory of Bifurcation, 3-46. In: Bardos, C., and Besis, D., eds., Bifurcation Phenomena in Mathematical Physics and Related Topics.

5. LALOUX, B.A. and MAWHIN, J., Multiplicity, Leray-Schauder Formula and Bifucation. J. Differential Equations 24 (1977) 309-322.

6. GAINES, R.E., and MAWHIN, J.L., Coincidence Degree and Nonlinear Differentia1 Equation. Springer-Verlag, Lecture Notes No. 568, 1977.

7. MA, T.W., Topological Degree of Set-values Compact Fields in Locally Convex Spaces. Dissertationes Mathematicae (Rosprawy Math.) XCII, 1972.

8. PFTRYSHYN, W.V., and FITZPATRICK, P.M., A Degree Theory, Fixed Point Theorems and Mapping Theorems for lultivalued Noncompact Nappings. Trans. Amer. Math. Soc. 194 (1974) 1-25.

9. ROCKAFELlER, R. Tyrre11, Integral Functionals, ioormal Integrands and Measurable Selections. 157-207, Non1inear Operators and the Calculus of Variations. Lecture Notes in Mathematics No. 543, Springer-Verlag. 1976. 


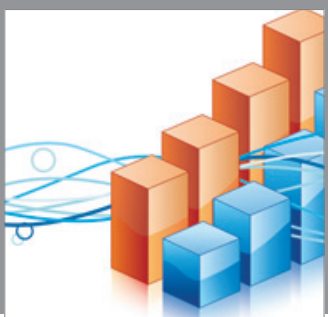

Advances in

Operations Research

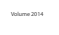

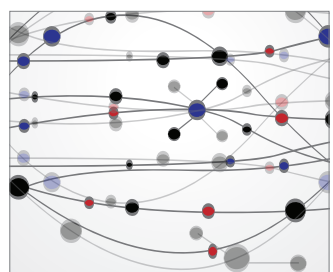

\section{The Scientific} World Journal
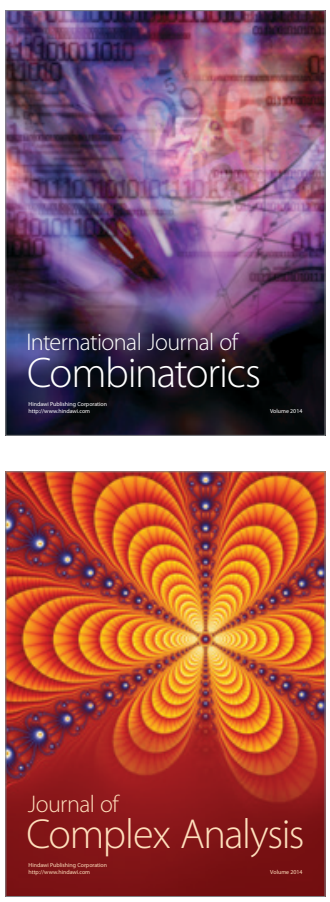

International Journal of

Mathematics and

Mathematical

Sciences
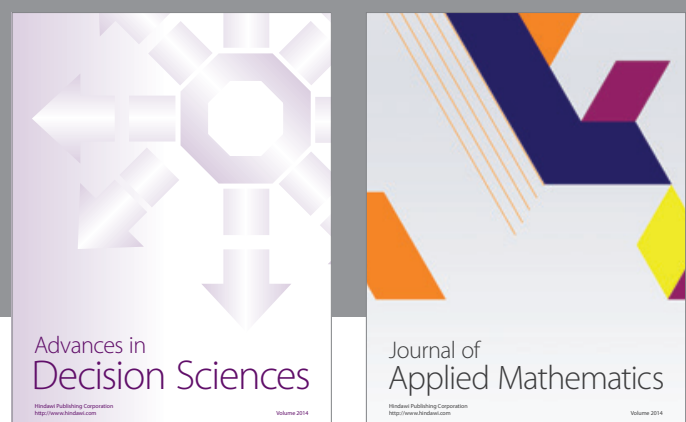

Journal of

Applied Mathematics
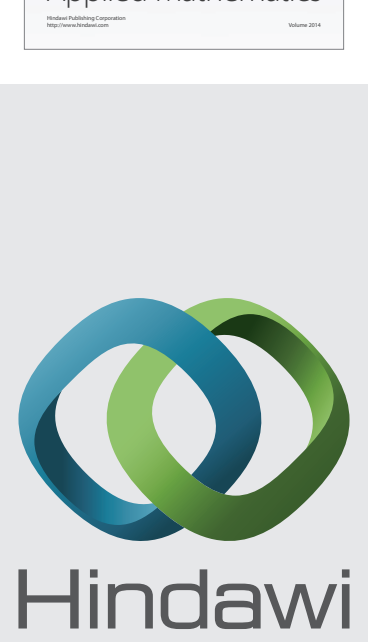

Submit your manuscripts at http://www.hindawi.com
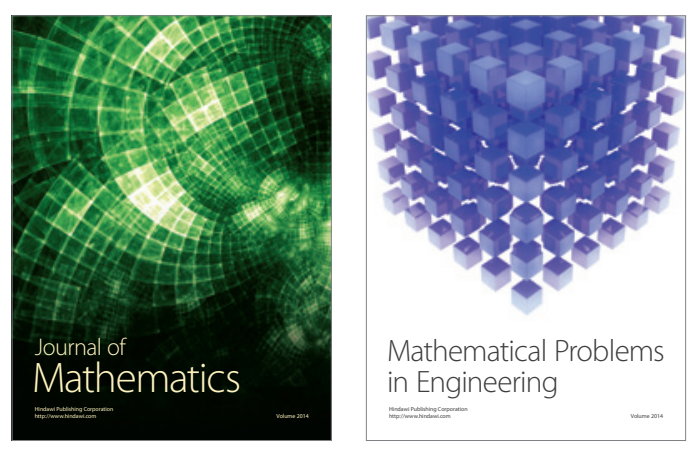

Mathematical Problems in Engineering
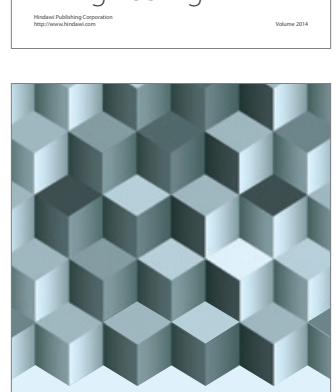

Journal of

Function Spaces
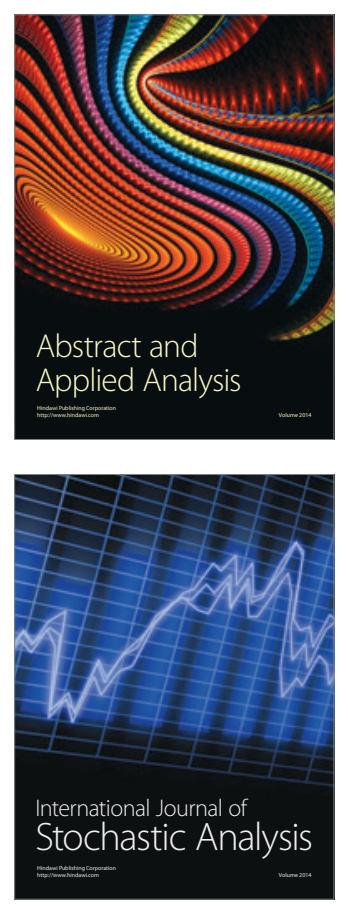

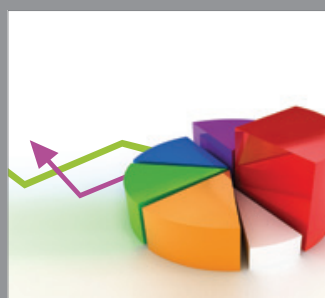

ournal of

Probability and Statistics

Promensencen
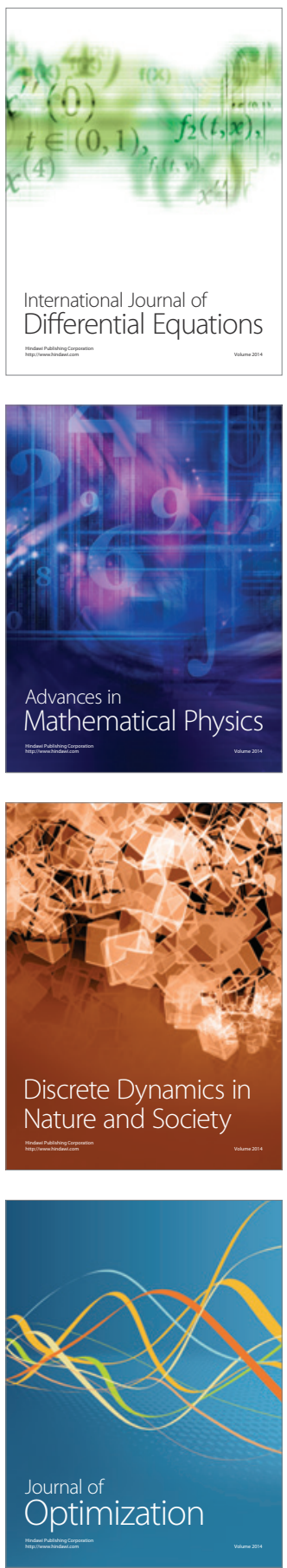